\title{
Testing the hot-flasher scenario with asteroseismological tools. First Results
}

\author{
M. M. Miller Bertolami ${ }^{1,2}$, A. H. Córsico ${ }^{1,2}$ and L. G. Althaus ${ }^{1,2}$ \\ ${ }^{1}$ Facultad de Ciencias Astronómicas y Geofísicas, UNLP, B1900FWA, La Plata, Argentina \\ ${ }^{2}$ Instituto Astrofísica La Plata, CCT-La Plata, UNLP-CONICET \\ email: mmiller@f caglp.unlp.edu.ar
}

\begin{abstract}
A core helium flash after the departure from the red giant branch (i.e. "hot-flasher scenario") offers one of the most promising explanations for the origin of He-sdO stars. Recently, Miller Bertolami et al. (2008) have shown that many surface properties of H-deficient sdO stars (the He-sdO stars) could be explained through this scenario if chemical diffusion is taken into account. In this context the He-sdO stars formed during a hot-flasher event would transform into H-rich hot-sdB stars (33000-38000 K) as a consequence of diffusion of the remaining $\mathrm{H}$ towards the surface of the star. Thus, some hot sdBs might be the descendants of He-sdO stars that have previously burnt most of their $\mathrm{H}$-content and, thus, a very thin $\mathrm{H}$ envelope should be expected $\left(10^{-9}\right.$ to $10^{-10} \mathrm{M}_{\odot}$, see Miller Bertolami et al. 2008 for details). Interestingly enough, the location of these $\mathrm{sdB}$ stars in the $\log g-T_{\text {eff }}$ diagram should overlap with the domain of the rapidly pulsating (p-mode) EC 14026 stars. This fact opens the interesting possibility of employing asteroseismology to investigate the existence of hot-sdB stars characterized by such very thin $\mathrm{H}$ envelopes. In this preliminary investigation, we explore the sensitivity of the acoustic pulsation spectrum of EC 14026 stars to the thickness of the H envelope.

The pulsation analysis presented in this work was performed with the help of the adiabatic radial and nonradial pulsation code employed by our group in numerous asteroseismological studies of white dwarfs and pre-white dwarfs (see Córsico et al. 2008 and references therein). The stellar models adopted in the present study where extracted from the $0.48150 M_{\odot}(\mathrm{Z}=0.001)$ sequence of Miller Bertolami et al. (2008). Given the exploratory nature of this work, in order to analyze the effects of different thicknesses of the $\mathrm{H}$-rich envelope on the pulsation spectrum of sdB stars we have artificially added a H-rich envelope in the outermost layers of our initial model. We have considered four different thicknesses of the $\mathrm{H}$ envelope $\left(\log M_{\text {env }} / \mathrm{M}_{\odot} \sim-4,-6,-8,-10\right)$ in addition to the self-consistent model (which lacks a $\mathrm{H}$ envelope). Next, we have pursued the evolution of the five sequences during the evolution on the HB. For each stellar model, we have computed the radial and nonradial p-modes with periods longer than 20 seconds, thus comfortably covering the observed period range of EC 14026 stars $(80-400 \mathrm{sec})$.

Our results show that the cycle of trapping is markedly smaller for the case of thick H-envelope models $\left(M_{\text {env }}>10^{-7} \mathrm{M}_{\odot}\right)$ than for the thin H-envelope models $\left(M_{\text {env }}<10^{-7} \mathrm{M}_{\odot}\right)$. We find that $\mathrm{sdB}$ stars with very thin $\mathrm{H}$ envelopes $\left(M_{\mathrm{env}}<10^{-7} \mathrm{M}_{\odot}\right)$ would not display almost any kind of trapping features in their frequency distribution at the observed range in EC 14026 stars $(\nu<13 \mathrm{mHz})$. Consequently their frequency spectra should be significantly different from that of normal sdB stars. We plan to explore in future works to which extent the shape of the chemical transitions (here adopted as simple gaussian profiles) affects the mode-trapping features.
\end{abstract}

Keywords. stars: evolution, stars: horizontal-branch, stars: subdwarfs, stars: oscillations.

\section{References}

Miller Bertolami, M., M., Althaus, L. G., Unglaub, K. \& Weiss, A 2008, A\&A, 491, 253

Córsico, A. H., Althaus, L. G., Kepler, S. O., Costa, J. E. S., \& Miller Bertolami, M. M. 2008, A\&A, 478, 869 